\title{
ARTICLE
}

\section{The acute effects of nicotine on corticostriatal responses to distinct phases of reward processing}

\author{
Kainan S. Wang ${ }^{1,2}$, Maya Zegel ${ }^{1}$, Elena Molokotos ${ }^{1,3}$, Lauren V. Moran $\mathbb{D}^{1,2}$, David P. Olson ${ }^{1,2}$, Diego A. Pizzagalli (D) ${ }^{1,2}$ and Amy C. Janes ${ }^{1,2}$
}

Nicotine enhances the reinforcement of non-drug rewards by increasing nucleus accumbens (NAcc) reactivity to anticipatory cues. This anticipatory effect is selective as no clear evidence has emerged showing that nicotine acutely changes reward receipt reactivity. However, repeated rewarding experiences shift peak brain reactivity from hedonic reward outcome to the motivational anticipatory cue yielding more habitual cue-induced behavior. Given nicotine's influence on NAcc reactivity and connectivity, it is plausible that nicotine acutely induces this shift and alters NAcc functional connectivity during reward processing. To evaluate this currently untested hypothesis, a randomized crossover design was used in which healthy non-smokers were administered placebo and nicotine (2-mg lozenge). Brain activation to monetary reward anticipation and outcome was evaluated with functional magnetic resonance imaging. Relative to placebo, nicotine induced more NAcc reactivity to reward anticipation. Greater NAcc activation during anticipation was significantly associated with lower NAcc activation to outcome. During outcome, nicotine reduced NAcc functional connectivity with cortical regions including the anterior cingulate cortex, orbitofrontal cortex, and insula. These regions showed the same negative relationship between reward anticipation and outcome as noted in the NAcc. The current findings significantly improve our understanding of how nicotine changes corticostriatal circuit function and communication during distinct phases of reward processing and critically show that these alterations happen acutely following a single dose. The implications of this work explain nicotinic modulation of general reward function, which offer insights into the initial drive to smoke and the subsequent difficulty in cessation.

Neuropsychopharmacology (2020) 45:1207-1214; https://doi.org/10.1038/s41386-020-0611-5

\section{INTRODUCTION}

Nicotine is a highly addictive naturally-occurring stimulant and the primary psychoactive ingredient in tobacco cigarettes [1]. It is reinforcing due to its ability to potentiate dopaminergic transmission in the nucleus accumbens [NAcc; 2, 3], a key node in the neural reward circuit $[4,5]$. In particular, a single acute dose of nicotine can induce an increase in mesolimbic dopamine (DA) activity to the NAcc $[3,6]$, resulting in increased reward-related learning $[7,8]$ and reward-seeking behavior [e.g., conditioned placed preference; 9-13]. These findings collectively highlight nicotine's ability to enhance DA function in reward-related brain regions, which subsequently influence how regions such as the NAcc respond to reward.

Prior work suggests that reward processing is separable into two linked but dissociable phases-reward anticipation and outcome [14]. Specifically, reward anticipation is motivated by the incentive salience of a reward-predictive cue or how much a reward is wanted [15-18], whereas reward outcome is associated with the pleasure of reward receipt or how much a reward is liked [19-22]. A recent meta-analysis of human functional magnetic resonance imaging (fMRI) studies confirmed that the NAcc responds to both anticipation and outcome phases [23]. Nicotine can influence such NAcc responding, given that, relative to placebo, acute nicotine induces a robust increase in NAcc reactivity to reward anticipation [24, 25]. Interestingly, how nicotine influences NAcc reactivity to reward outcome is not well documented and the few studies that have explicitly examined this have reported no significant effect of nicotine on NAcc activity during reward outcome $[24,26]$, suggesting that nicotine preferentially affects reward anticipation. The focus of nicotine's effect on reward anticipation, but not outcome, is likely driven by how nicotine influences the NAcc, which is to effectively increase DA transmission and the incentive salience encoding of rewarding cues during anticipation [27-29].

This increase in NAcc activity towards reward anticipation under nicotine is in line with animal models showing that nicotine facilitates the shift in the NAcc signal from hedonic encoding of a primary reward to the motivational encoding of the cue associated with the reward [30-33]. This prompts the question of whether the observed increase in NAcc activity during reward anticipation is accompanied by a simultaneous decrease in NAcc activity towards reward outcomes. That is, does a single acute dose of nicotine swing the balance of NAcc activity towards responding to the incentive salience of a reward cue (i.e., anticipation) at the expense of responding to the hedonic drive of the reward itself (i.e., outcome)? If so, a related question is whether this shift in NAcc activity under nicotine could subserve changes in how the NAcc is communicating with other rewardprocessing foci in the cortex. Prior work has showed that nicotine administration can impact the firing patterns in cortical regions

\footnotetext{
${ }^{1}$ McLean Imaging Center, McLean Hospital, Belmont, MA, USA; ${ }^{2}$ Harvard Medical School, Boston, MA, USA and ${ }^{3}$ Department of Psychology, Suffolk University, Boston, MA, USA Correspondence: Amy C. Janes (ajanes@mclean.harvard.edu)
}

Received: 30 October 2019 Revised: 6 January 2020 Accepted: 7 January 2020

Published online: 13 January 2020 
and the NAcc $[22,34,35]$, thereby diminishing the ability of the cortex to exert top-down influence on the NAcc during reward-seeking behaviors [for review see 36-38]. Given that exposure to nicotine can disrupt the corticostriatal circuit, it is likely that nicotine's influence on the NAcc has a direct consequence on how this particular reward node is functionally connected to key reward-related cortical regions during reward processing.

Here, we investigated these questions by taking advantage of the monetary incentive delay (MID) task $[39,40]$, which allows for the dissociation of reward anticipation and outcome. Using a within-subject study design with healthy non-smoking individuals, we were specifically interested in testing whether acute nicotine administration, relative to placebo, induces a negative correlation between NAcc activity during reward anticipation and outcome and whether nicotine induces changes in task-dependent corticostriatal connectivity.

\section{METHODS}

Participants

Seventeen (8 females) participants (mean age: $26.06 \pm 6.09$ ) were recruited as part of the healthy control group for another published study [41]. Participants were self-reported non-smokers with less than 20 lifetime uses of nicotine, denied nicotine use in the past year, and had expired carbon monoxide (CO) level of less than $5 \mathrm{ppm}$ at each study visit. Participants were administered the structured clinical interview for DSM-IV-TR [42] to screen for lifetime history or current diagnosis of any of the following psychiatric illnesses: organic mental disorder, schizophrenia, schizoaffective disorder, delusional disorder, psychotic disorders not otherwise specified, bipolar disorder, attention deficit hyperactivity disorder (ADHD), mood congruent or incongruent psychotic features, generalized anxiety disorder and social anxiety disorder. In addition, participants were excluded if they met any of the following criteria: failure to meet MRI safety requirements, lifetime history of electroconvulsive therapy, anticholinergic drug use in the past week, history or current cardiac problems such as known arrhythmia, acute coronary syndromes, or ischemic heart disease, seizure disorder, psychotropic medication or illicit substance use, and breath blood alcohol levels greater than zero. Furthermore, participants provided urine samples to confirm that they were not pregnant or had not engaged in recent drug use, both of which were contraindicative to study participation. Participants provided written informed consent in accordance with the experimental protocol approved by the Partners HealthCare Institutional Review Board upon receiving a complete description of the experiment.

\section{Study drug}

Participants were administered nicotine (Nicorette Lozenge, GlaxoSmithKline, Brentford London) and placebo (Tums antacid, GlaxoSmithKline, Brentford London) lozenges on two separate study days roughly 1 week apart. The nicotine lozenge contained 2-mg of nicotine (mean half-life of $2.3 \mathrm{~h}$ ), which was predicted to reach a peak systemic nicotine concentration of $4.4 \mathrm{ng} / \mathrm{ml}$ in approximately $1 \mathrm{~h}$ after administration [43]. While pharmacokinetic profiles differ between tobacco smoking and nicotine lozenges, the lozenge dose chosen delivers the total amount of nicotine typically received from smoking a single cigarette [44-46]. Drug administration was conducted in a randomized, counterbalanced, and double-blind manner. In particular, efforts were made to ensure that there were no physical (same size, shape, and color) or gustatory (both mint-flavored) differences between the lozenges. On both study days, $1 \mathrm{~h}$ prior to entering the fMRI scanner, participants were instructed to place the lozenge directly into their mouth, next to their cheek and allow it to dissolve without chewing (which took roughly $15 \mathrm{~min}$ ).
We monitored participants for any potential side effect following ingestion of the lozenges. However, we did not probe for any subjective pleasurable effects. Although we do not preclude the possibility that there are nuanced differences in how the lozenges tasted, no participant reported distinct sensations while taking either lozenge. Systemic nicotine presence in participants on both study days were verified by measuring cotinine, a reliable biomarker for nicotine absorption [47].

MID task

Similar to prior work [39], we used a MID task that features both anticipation and outcome phases. Briefly, each trial was divided into cue anticipation, target response, and outcome presentation phases. The cue anticipation ( $0.5 \mathrm{~s}$ ) and target response $(0.15 \mathrm{~s})$ phases were separated by a variable inter-stimulus interval $(2.25-3.75 \mathrm{~s})$ and the target response and outcome presentation $(1.25 \mathrm{~s})$ phases were separated by another inter-stimulus interval $(2.4-3.9 \mathrm{~s})$. Each trial ended with a variable inter-trial interval lasting (1.50-4.5 s). Each of the cues presented during the anticipation phase (reward: +\$; neutral: 0\$; punishment: $-\$$ ) was, respectively, associated with feedback during the outcome phase denoting whether participants received monetary gain $(+$ $\$ 1.96$ to $+\$ 2.34)$, monetary loss $(-\$ 1.81$ to $-\$ 2.19)$ or no-change (\$0). During the target response phase, participants were told to respond as quickly as possible to the displayed red square so as to maximize their chances of earning rewards and avoiding punishments. To equalize task difficulty across participants, each participant's 70th percentile reaction time in the practice session was defined as their individual success threshold. The task consisted of five blocks of 24 trials ( 8 reward, 8 neutral and 8 punishment cues) and participants were not informed of cumulative earnings during the task. Our main conditions of interest were the anticipation and outcome phases for both reward and neutral trials.

Neuroimaging data collection and preprocessing

Images were collected using a Siemens Trio 3T scanner (Erlangen, Germany) with a 32-channel head coil. Multiecho multi-planar rapidly acquired gradient echo-structural images (MPRAGE) were acquired with the following parameters $(T R=2.1 \mathrm{~s}, \mathrm{TE}=3.3 \mathrm{~ms}$, slices $=128$, matrix $=256,256$, flip angle $7^{\circ}$, resolution $1.0 \times 1.0 \times$ $1.33 \mathrm{~mm}$ ). Slices for the functional images were acquired aligned to the anterior and posterior commissures and the phase encode direction was set from the posterior to anterior direction to prevent prefrontal signal loss. A multi-band acquisition was used for the functional images with the following parameters $(T R=$ $0.72 \mathrm{~s}, \mathrm{TE}=0.32 \mathrm{~s}$, multi-band acceleration factor $=6$, flip angle $66^{\circ}$, slices $=54$, voxel size $=3 \times 3 \times 3 \mathrm{~mm}$ ).

Images were processed using fMRI of the Brain (FMRIB) Software Library (FSL; www.fmri-b.ox.ac.uk/fsl). In particular, we first brain extracted the images using BET and corrected for motion using MCFLIRT. We subsequently coregistered the mean functional image to the anatomical scan and normalized the anatomical to standard space. The normalized anatomical image was then used to reslice the functional data to standard stereotaxic space defined by the Montreal Neurological Institute (MNI). We also applied slice timing correction and spatial smoothing at full-width halfmaximum of $6 \mathrm{~mm}$ to the normalized functional data.

\section{Neuroimaging analyses}

We conducted two general linear models (GLMs) to examine changes in neural activity in response to acute nicotine administration and to evaluate differences in task-based connectivity. Details pertaining to each GLM are given in subsequent sections. In both GLMs, we included six confound regressors for the $x, y, z$ translational and rotational motion. To further address motion, we identified timepoints representing motion and intensity artifacts (https://github.com/bbfrederick/spikefix) and 
added a regressor to remove these timepoints in the model. All task regressors were convolved with the canonical hemodynamic response function. In the second-level analysis, we combined each participant's five task runs using a fixed-effects model. Finally, in the group-level analysis, we carried out a mixed-effects model with FLAME $1+2$ to combine data across all participants. Flame $1+2$ fits the model using Bayesian modeling for mixed-effects variance estimation before processing the voxels that were near threshold with Metropolis-Hastings Markov Chain Monte Carlo sampling to obtain a more precise estimation of the mixed-effect variance [48]. Except where noted, all z-statistics images were thresholded and corrected for multiple comparisons using an initial cluster-forming threshold of $z>3.1$ followed by a conservative whole-brain cluster-extent threshold of $p<0.05$, as determined by Gaussian Random Field Theory [49, 50]. Images were created using MRIcroGL (https://www.nitrc.org/ projects/mricrogl) and overlaid on the MNI152 T1 brainextracted brain.

\section{Standard GLM}

Our first GLM was designed to evaluate nicotine's effects on reward-related activation. We individually modeled the five task runs for each participant in the first-level analysis. Each first-level GLM consisted of six task regressors representing reward cue, neutral cue, punishment cue, reward outcome, neutral outcome, and punishment outcome, respectively. Our main contrasts of interest were between the reward and neutral conditions during both the anticipation (i.e., cue) and outcome phases.

We applied a small-volume correction restricting our region of interest to the bilateral anatomical NAcc. As detailed in the introduction, we focused on the NAcc to determine: (1) if we can replicate prior work showing that, relative to placebo, nicotine increases NAcc activity during reward anticipation and (2) whether nicotine alters the relationship between NAcc activity during reward anticipation and outcome. For the second question, we extracted the NAcc parameter estimates and correlated them between the two reward phases. We then performed a fisher $r$-to- $z$ transformation on the correlation coefficients for the placebo and nicotine visits, respectively, and directly compared them to assess for significant differences.

\section{Psychophysiological interaction (PPI) GLM}

In our second GLM, we implemented a PPI model using an anatomical region of interest in the bilateral NAcc as the seed region to create the physiological regressor. We also added in the same task regressors used for the first standard GLM. To form the PPI regressors, we multiplied the physiological regressor by the convolved task regressor modeling either the reward cue or outcome. In the group-level analysis, we conducted a withinsubject two-sample paired $t$-test to compare the nicotine and placebo PPI regressors for both anticipation (i.e., reward cue) and outcome phases, respectively. In addition, to determine whether NAcc activation to anticipation impacted functional connectivity during the outcome, we also added each participant's mean NAcc parameter estimates for placebo and nicotine visits, respectively, as covariates in the group-level two-sample $t$-test for the outcome PPI regressor.

\section{RESULTS}

Cotinine levels between visits

A paired-sample $t$-test revealed a significant elevation in participants' serum cotinine levels during the nicotine visit compared to the placebo visits $(t(15)=2.78, p=0.014)$. One participant could not provide a blood sample on the placebo visit and thus was removed from this particular analysis. However, urinalysis was used in this case to confirm the absence of cotinine. functional magnetic resonance imaging (fMRI)

NAcc activity between placebo and nicotine during reward anticipation. First, we were interested in replicating our prior work in an independent sample [25] where participants showed significantly greater NAcc activity to reward anticipation when they were administered nicotine compared to placebo. We conducted a two-sample paired $t$-test with a small-volume correction using an anatomical bilateral NAcc mask [41]. Replicating prior findings, participants showed significantly stronger NAcC activation during the nicotine compared to placebo visit (Fig. 1; peak $z$-stat $=3.78$ at $\mathrm{MNI}_{x, y, z}=12,18,-6$, $p_{\text {corr }}=0.031$ ). Also in line with prior reports, no activation was significant when comparing nicotine and placebo during reward outcome.

Relationship between NAcc activity during reward anticipation and outcome under nicotine. Next, we examined whether acute administration of nicotine changed the relationship between NAcc responses to reward anticipation and outcome. In other words, did nicotine alter the association between NAcc activity to anticipation and its activity to outcome? To evaluate this, we applied an anatomical bilateral NAcc mask and extracted the mean parameter estimate (PE) for each participant from both the reward-neutral cue (i.e., anticipation) and rewardneutral outcome (i.e., outcome) contrasts. We correlated participants' anticipation and outcome NAcc activity within each visit (e.g., nicotine or placebo) and found a significant negative relationship in the nicotine visit (Fig. $2 \mathrm{~b} ; r=-0.57 ; p=$ 0.018 ) that was absent in the placebo visit (Fig. $2 a ; r=0.25, p=$ $0.33)$. These correlations were significantly different $(Z=-2.39$, $p=0.017$ ).

Corticostriatal connectivity during reward anticipation and outcome under nicotine. In our PPI model, we used an anatomical bilateral NACC as our seed region to identify regions whose connectivity with the NAcc increases during reward anticipation or outcome phases. We directly compared connectivity changes under placebo relative to nicotine administration using a twosample paired $t$-test. For the placebo-nicotine contrast, we found a cluster within the anterior cingulate cortex (ACC) extending into the anterior midcingulate cortex (aMCC; Fig. 1b; peak $z$-stat $=3.15$ at $\mathrm{MNI}_{x, y, z}=4,-4,50 p_{\text {corr }}=0.0029$ ) exhibited significantly greater connectivity with the NAcc during the outcome phase under placebo relative to nicotine (contrast of placebo-nicotine). No differences in functional connectivity were noted when assessing nicotine administration vs. placebo (contrast of nicotine-placebo). No contrasts for the anticipation phase survived correction.

To further examine the effect of nicotine on corticostriatal functional connectivity during the outcome phase, we included each participant's NAcc PE during anticipation as a covariate into the PPI model. The inclusion of the covariate into the PPI model allowed us to test for regions whose connectivity with NAcc increases during the outcome phase as a function of their NACC activity during the anticipation phase. For the contrast of placebo-nicotine, after accounting for NAcc anticipation as a covariate, we found a significant cluster in the orbitofrontal cortex (OFC), insula, and ACC (Fig. 1c; peak z-stat $=3.75$ at $\mathrm{MNI}_{x}$, $\left.y, z=-32,20,-2, p_{\text {corr }}=0.0098\right)$.

Relationship between cortical activity during reward anticipation and outcome under nicotine. Given the significant findings from our PPI models, we conducted a post hoc exploratory analysis to probe whether these cortical regions also exhibited the negative relationship between reward anticipation and outcome emerging for the NAcc. For each of the three cortical regions (i.e., ACC, insula and OFC), we created a spherical functional mask extending $5 \mathrm{~mm}$ from the peak voxel. Next, we extracted the 

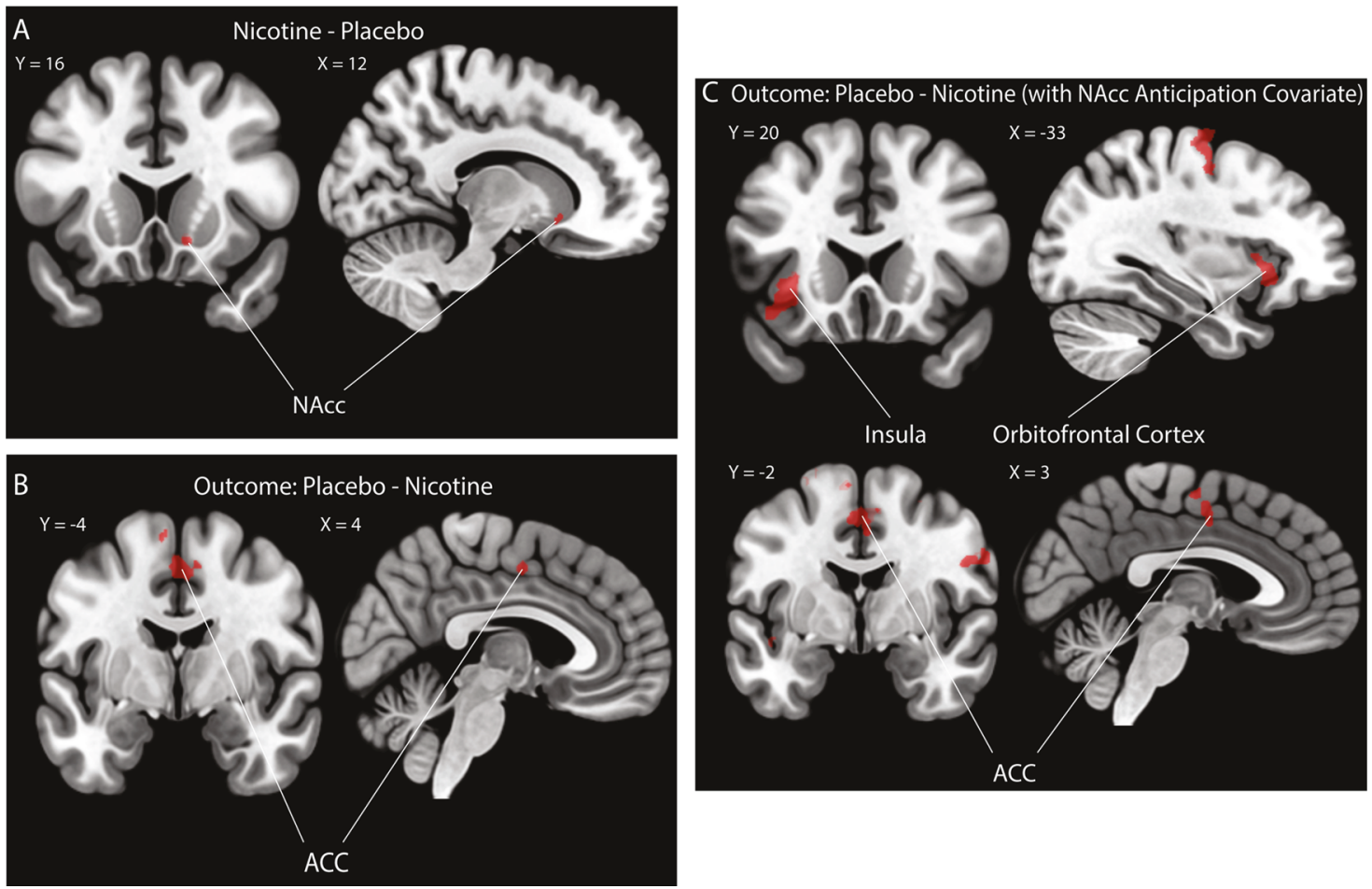

Fig. 1 Effect of nicotine on corticostriatal activity to reward processing. a NAcc activity between nicotine and placebo visits. Acute nicotine administration significantly increased participants' NAcc activity during reward anticipation. All voxels passed an initial cluster-forming threshold of $z=3.1$, with small-volume correction within the anatomical NAcc using a cluster-extent threshold of $p<0.05$. $\mathbf{b}$ PPI analysis of functional connectivity between NAcc and other brain regions. Acute nicotine administration significantly decreased the functional connectivity between NACC and the ACC/MCC during reward outcome but not anticipation. c After adding in participants' NAcc activation during reward anticipation as a covariate, the PPI model revealed that acute nicotine administration significantly decreased the functional connectivity between NAcc and three cortical regions (i.e., ACC, OFC and insula) during the reward outcome phase. All areas of activation passed an initial cluster-forming threshold of $z=2.3$, with whole-brain cluster-extent threshold at $p<0.05$.
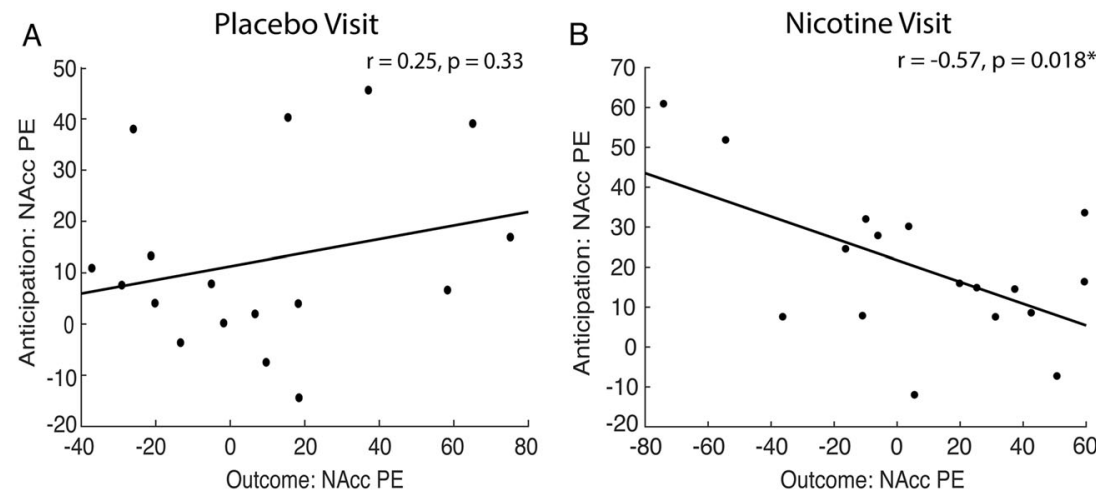

Fig. 2 NAcc anticipation vs outcome relationship between visits. a In the placebo visit, participants' NAcc activity during reward anticipation and outcome did not show any significant relationship. b Following acute nicotine administration, participants' NAcc activity demonstrated a significant negative relationship between the reward anticipation and outcome phases. There was a significant difference in the two correlation coefficients for the placebo and nicotine visits.

mean activation from the functional masks for both the anticipation (reward-neutral cue contrast from first GLM) and outcome (reward-neutral outcome contrast from first GLM) phases. We then correlated the activity between the two phases for each ROI.

We found that neither the ACC (Fig. 3a; $r=-0.22, p=0.39$ ), insula (Fig. 3c; $r=-0.47, p=0.055$ ), nor the OFC (Fig. 3e; $r=0.44, p=0.075)$ showed a significant relationship in their activity between the two phases under placebo. Following acute nicotine administration, the ACC (Fig. 3b; $r=-0.71$, $p=0.0015$ ) and the OFC (Fig. $3 d ; r=-0.49, p=0.045)$ both showed significant negative correlations in their activity between the two phases. The insula did not initially show a significant negative correlation between reward phases $(r=$ $0.088, p=0.74$ ) but after removal of a statistical outlier, its relationship became significant as well (Fig. 3f; $r=-0.50, p=$ 0.049). Comparing the correlation coefficients between visits (i.e., a one-tailed test for nicotine visit coefficient being more negative than placebo visit) for each cortical ROI, we found that the ACC $(z=1.76, p=0.039)$, OFC $(z=2.67, p=0.0038)$, and insula $(z=2.8, p=0.0026)$ all showed significantly different relationships between visits. 
A

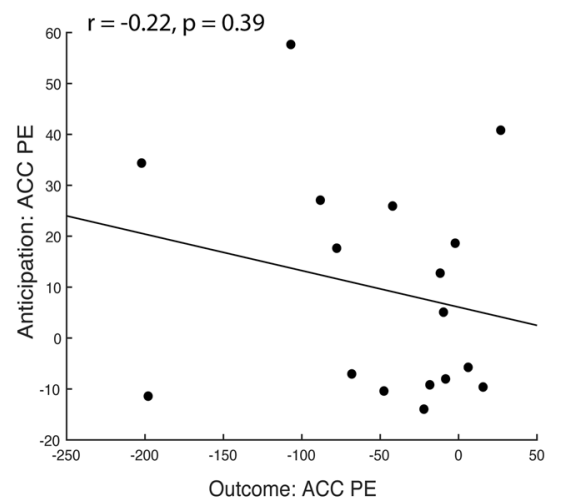

D

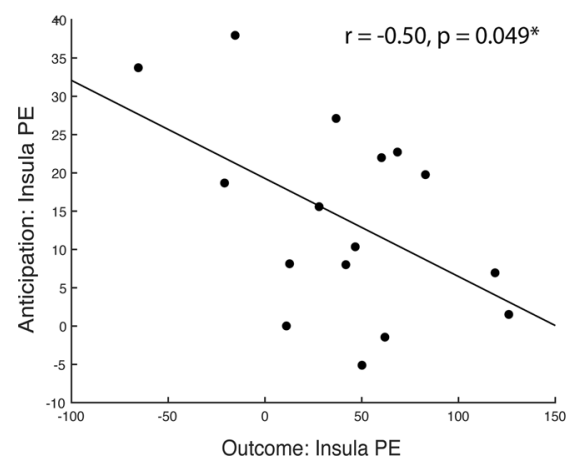

B

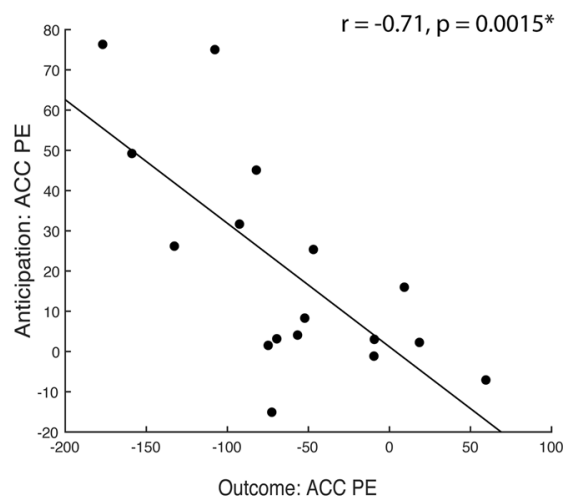

E

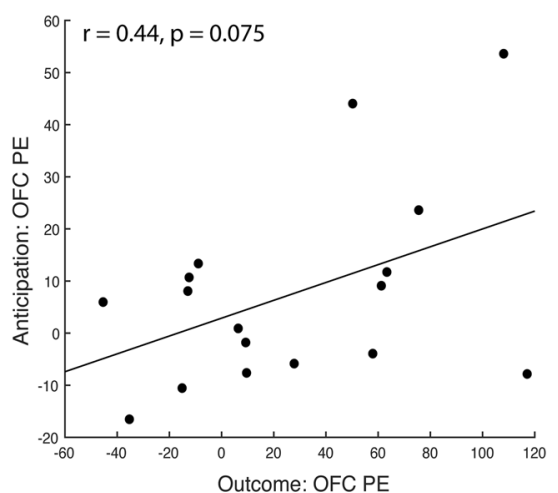

C

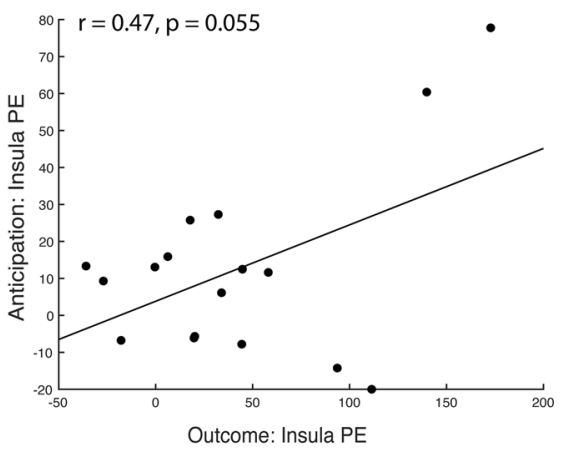

F

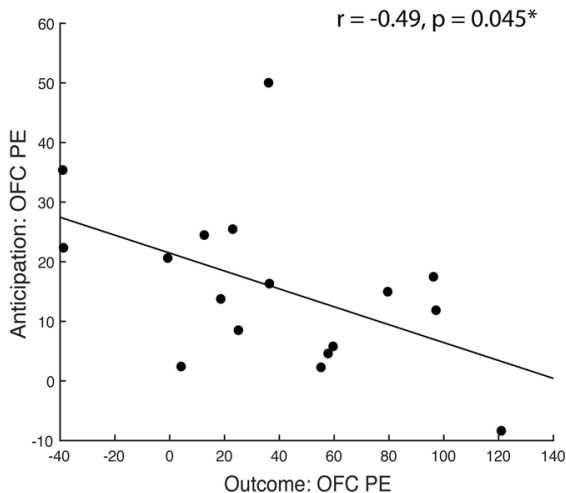

Fig. 3 Relationship between anticipation and outcome activity in the cortical regions of interest. a, $c$, e In the placebo visit, none of the three cortical regions of interest (i.e., ACC, insula, OFC) showed a significant relationship between their activity during reward anticipation and outcome. b, d, f Following acute nicotine administration, participants' respective activity in the ACC, insula, and OFC all demonstrated a significant negative relationship between the reward anticipation and outcome phases.

\section{DISCUSSION}

The current work shows that acute nicotine administration not only produced a significant increase in NAcc activity during reward anticipation, but more importantly, induced a significant negative relationship in NAcc activity between reward anticipation and outcome. This indicates that greater NAcc response towards anticipation is coupled with lower NAcc response towards reward outcome, indicating a shift in NAcc activity favoring reward anticipation over outcome following a single acute dose of nicotine in non-nicotine users.

Under drug-naïve conditions, the NAcc consistently responds to both reward anticipation and outcome, thereby subserving both the incentive salience of a reward-associated cue and the hedonic properties of the reward itself $[23,51,52]$. In contrast, under the influence of nicotine, the NAcc receives a heightened mesolimbic dopaminergic transmission that results in an increase in its activity towards conditioned cues during reward anticipation [53-56]. Confirming prior work [24, 25], we reported that nicotine significantly increased NAcC activity during anticipation to reward. Building on this observation, we showed that this increase in NAcc activity during anticipation is coupled with a corresponding decrease in NAcc activity during outcome. This significant negative relationship between NAcc activity during reward anticipation and outcome is masked in typical analysis methods since the current and prior work [24] has not observed changes in NAcc activity to outcome when nicotine and placebo visits were directly compared. This significant negative relationship in the nicotine visit suggests that nicotine is facilitating the shift in NAcc responding towards anticipation at the expense of outcome, which parallels the prediction error hypothesis of DA shifting its firing from the reward to the conditioned stimulus [for review see 57-59] and animal work demonstrating that exposure to nicotine increases the drug-seeking and drug-taking behaviors (i.e., wanting) of other drugs of abuse $[60,61]$. It is plausible that nicotine's potentiation of mesolimbic DA transmission to the NAcc subserves the shift in NAcc response from outcome to anticipation, thereby accelerating the cue's acquisition of incentive salience. The association between greater NAcc reactivity to anticipation and lower NAcc connectivity during outcome is reminiscent of the shift from goal-directed to habitual responding typically seen following chronic drug use [62-64]. What is notable is that this shift appears to happen not over repeated nicotine use but following a single 2-mg administration in non-smokers.

Further probing of NACC activity revealed that acute nicotine significantly decreased the functional connectivity between NAcc and ACC specifically during the outcome phase (discussed in detail in the next paragraph). Intriguingly, after accounting for NAcc anticipatory activation, two additional cortical regions (i.e., OFC and insula) also showed decreased functional connectivity with the NAcc during the outcome phase. These cortical regions have all been implicated as key players in the reward-processing circuit [for review see 65] and in particular, the OFC and insula are proposed as hedonic hotspots in the brain [14]. The role of these two cortical regions during reward processing is to monitor the stimulus features of the reward and translate this into a hedonic value that can be passed onto the NAcc for behavioral updates [66-68]. In other words, while acute nicotine does not blunt NAcc reactivity to reward outcome, it is plausible, with evidence from prior work reporting that participants pre-exposed to nicotine reported lower subjective "liking" of potent rewards such as 
cocaine [69], that nicotine may disrupt the hedonic drive of a rewarding stimulus (i.e., how pleasurable is the reward) by reducing communication within the reward pathway. As such, this ability for nicotine to acutely disrupt corticostriatal connectivity towards reward outcomes showcases nicotine's potent ability to perturb the reward-processing circuitry that can compound into long-term behavioral consequences. The extent of how nicotine alters the hedonic drive can be probed in future studies exploring nicotine-induced changes in the pleasurable sensation of consuming other drugs and rewards.

Of the three cortical regions showing nicotine-induced decrease in functional connectivity with NAcc, the most notable is ACC because it exhibited the same negative relationship as NAcc in its response to reward anticipation and outcome. Although the OFC and insula also showed this negative relationship, ACC had the strongest negative relationship that was comparable to that demonstrated by NAcc. Prior animal work has demonstrated that the ACC and NACC are functionally connected during reward processing [70-73] and that nicotine reduces the communication between ACC glutamatergic neurons and NAcc-bound VTA DA neurons $[37,74,75]$. In the same vein, our current finding in humans also points to a nicotine-induced reduction of ACC-NACC connectivity during reward outcome that may contribute to both regions showing a similar shift in their responses from reward outcome to anticipation. This latter observation is important given that both ACC and NACC are recipients of heavy dopaminergic projections from the ventral tegmental area that increases DA signaling under nicotine [76-80] and that both regions display response profiles matching the generation of prediction error during cue-outcome associative learning [81-86]. Therefore, it seems likely that the disruption of the functional connectivity between ACC and NACC during reward outcome is related to the negative relationship that both regions exhibited towards anticipation and outcome. Whether the former engenders the latter remains an open question for future research.

The current work includes the following limitations. First, even though our current sample size is comparable with previously published datasets applying similar methodology [e.g., 87], future studies should recruit a larger sample to replicate and extend our reported findings into domains such as sex differences. Second, our current study was designed to measure brain activation during the anticipated peak nicotine levels estimated across the population. However, it is plausible that nicotine's effect on this shift may differ between individuals depending on their rate of nicotine metabolism [88], suggesting the need for future research evaluating how metabolism influences the currently-presented data. It is also likely that nicotine's neural effect on general reward function and in particular, its abuse potential and transition to chronic use, is dependent on the route of intake and the associated pharmacokinetics. Thus, future studies should also evaluate how variance in pharmacokinetics due to different consumption methods (smoked vs. oral) influence the shift observed in the ACC and NAcc. Despite these limitations, the current work employed a rigorous design, which not only replicated the nicotine-induce facilitation of NAcc reactivity to reward anticipation, but revealed other strong effects supported by the existent literature.

Overall, our findings provide a more nuanced perspective on how nicotine impacts reward processing beyond its ability to enhance the motivational drive of anticipatory reward cues. The observation that a single acute dose of nicotine creates a shift in NAcc activity from outcome to anticipation expands on the traditional view of nicotine heightening incentive salience. Further, these findings were noted in non-smokers using a task involving monetary rewards, not stimuli previously associated with nicotine, thus fitting with the idea that nicotine has a global influence on reward processing [53]. Such influences on reward processing may not only contribute negatively towards smoking cessation but could also increase the susceptibility to dependence and difficulty quitting of other abused substances used concurrently with nicotine [89]. In addition, nicotine's ability to disrupt the corticostriatal communication specifically during the hedonicdriven outcome phase of reward processing hints at potential mood-related consequences, consistent with the high comorbidity between nicotine use and mood disorders [90] as well as the observed mood improvements following smoking cessation [91]. In short, these findings showcase the potent acute effect of nicotine on altering both the activity and the communication between key nodes in the reward-processing circuitry to powerfully drive adaptive behaviors.

\section{FUNDING AND DISCLOSURE}

This work was supported by funding from the National Institutes on Drug Abuse grants K10 DA029645, K02 DA042987 and R01 DA039135 (A.C.J.). D.A.P. was partially supported by R37 MH068376 from the National Institute of Mental Health. The content is solely the responsibility of the authors and does not necessarily represent the official views of the National Institutes of Health.

Over the past 3 years, DAP has received consulting fees from Akili Interactive Labs, BlackThorn Therapeutics, Boehringer Ingelheim, and Takeda Pharmaceuticals and an honorarium from Alkermes for activities unrelated to the current work. All other authors declare no competing financial interests.

\section{AUTHOR CONTRIBUTIONS}

A.C.J. conceptualized and designed the study with DAP. M.Z. recruited and collected the data. K.S.W. conducted all analyses and wrote the initial draft with A.C.J. and E.M. L.V.M. and D.O. provided clinical expertise and served as study physicians. All authors reviewed and edited the final manuscript.

\section{ADDITIONAL INFORMATION}

Publisher's note Springer Nature remains neutral with regard to jurisdictional claims in published maps and institutional affiliations.

\section{REFERENCES}

1. Stolerman $\mathbb{I P}$, Jarvis $M$. The scientific case that nicotine is addictive. Psychopharmacology. 1995;117:2-10.

2. Pontieri FE, Tanda G, Orzi F, Di Chiara G. Effects of nicotine on the nucleus accumbens and similarity to those of addictive drugs. Nature. 1996;382:255.

3. Rice ME, Cragg SJ. Nicotine amplifies reward-related dopamine signals in striatum. Nat Neurosci. 2004;7:583.

4. Wang KS, Smith DV, Delgado MR. Using fMRI to study reward processing in humans: past, present, and future. J Neurophysiol. 2016;115:1664-78.

5. Delgado MR. Reward-related responses in the human striatum. Ann N Y Acad Sci. 2007;1104:70-88.

6. Zhang T, Zhang L, Liang Y, Siapas AG, Zhou F-M, Dani JA. Dopamine signaling differences in the nucleus accumbens and dorsal striatum exploited by nicotine. $J$ Neurosci. 2009;29:4035-43.

7. Barr RS, Pizzagalli DA, Culhane MA, Goff DC, Evins AE. A single dose of nicotine enhances reward responsiveness in nonsmokers: implications for development of dependence. Biol Psychiatry. 2008;63:1061-5.

8. Olausson P, Jentsch JD, Taylor JR. Nicotine enhances responding with conditioned reinforcement. Psychopharmacology. 2004;171:173-8.

9. Collins AL, Aitken TJ, Greenfield VY, Ostlund SB, Wassum KM. Nucleus accumbens acetylcholine receptors modulate dopamine and motivation. Neuropsychopharmacology. 2016;41:2830.

10. Sun N, Laviolette SR, Group AR. Dopamine receptor blockade modulates the rewarding and aversive properties of nicotine via dissociable neuronal activity patterns in the nucleus accumbens. Neuropsychopharmacology. 2014;39:2799.

11. Jerlhag E, Engel JA. Ghrelin receptor antagonism attenuates nicotine-induced locomotor stimulation, accumbal dopamine release and conditioned place preference in mice. Drug Alcohol Depend. 2011;117:126-31. 
12. Spina L, Fenu S, Longoni R, Rivas E, Di Chiara G. Nicotine-conditioned single-trial place preference: selective role of nucleus accumbens shell dopamine D 1 receptors in acquisition. Psychopharmacology. 2006;184:447-55.

13. Walters $\mathrm{CL}$, Cleck JN, Kuo Y-C, Blendy JA. $\mu$-Opioid receptor and CREB activation are required for nicotine reward. Neuron. 2005;46:933-43.

14. Olney JJ, Warlow SM, Naffziger EE, Berridge KC. Current perspectives on incentive salience and applications to clinical disorders. Curr Opin Behav Sci. 2018;22:59-69.

15. Ostlund SB, LeBlanc KH, Kosheleff AR, Wassum KM, Maidment NT. Phasic mesolimbic dopamine signaling encodes the facilitation of incentive motivation produced by repeated cocaine exposure. Neuropsychopharmacology. 2014;39:2441.

16. Peciña S, Berridge KC. Dopamine or opioid stimulation of nucleus accumbens similarly amplify cue-triggered 'wanting'for reward: entire core and medial shell mapped as substrates for PIT enhancement. Eur J Neurosci. 2013;37:1529-40.

17. Hickey C, Peelen MV. Neural mechanisms of incentive salience in naturalistic human vision. Neuron. 2015;85:512-8.

18. Berridge KC, Robinson TE. Liking, wanting, and the incentive-sensitization theory of addiction. Am Psychologist. 2016;71:670.

19. Smith KS, Mahler SV, Peciña S, Berridge KC. Hedonic hotspots: generating sensory pleasure in the brain. In: Kringelbach ML, Berridge KC (eds). Pleasures of the brain. New York, NY: Oxford University Press; 2010. p. 27-49.

20. Smith KS, Berridge KC. The ventral pallidum and hedonic reward: neurochemical maps of sucrose "liking" and food intake. J Neurosci. 2005;25:8637-49.

21. Mahler SV, Smith KS, Berridge KC. Endocannabinoid hedonic hotspot for sensory pleasure: anandamide in nucleus accumbens shell enhances 'liking'of a sweet reward. Neuropsychopharmacology. 2007;32:2267.

22. Castro DC, Berridge KC. Opioid hedonic hotspot in nucleus accumbens shell: $\mathrm{mu}$, delta, and kappa maps for enhancement of sweetness "liking" and "wanting". J Neurosci. 2014;34:4239-50.

23. Oldham S, Murawski C, Fornito A, Youssef G, Yücel M, Lorenzetti V. The anticipation and outcome phases of reward and loss processing: a neuroimaging meta-analysis of the monetary incentive delay task. Hum Brain Mapp. 2018; 39:3398-418.

24. Rose EJ, Ross TJ, Salmeron BJ, Lee M, Shakleya DM, Huestis MA, et al. Acute nicotine differentially impacts anticipatory valence-and magnitude-related striatal activity. Biol. Psychiatry. 2013;73:280-8.

25. Moran LV, Stoeckel LE, Wang K, Caine CE, Villafuerte R, Calderon V, et al. Nicotine increases activation to anticipatory valence cues in anterior insula and striatum. Nicotine Tob Res. 2017;20:851-8.

26. Bühler M, Vollstädt-Klein S, Kobiella A, Budde $H$, Reed $\sqcup$, Braus DF, et al. Nicotine dependence is characterized by disordered reward processing in a network driving motivation. Biol Psychiatry. 2010;67:745-52.

27. Berridge KC, Robinson TE. What is the role of dopamine in reward: hedonic impact, reward learning, or incentive salience? Brain Res Rev. 1998; 28:309-69.

28. Berridge KC. The debate over dopamine's role in reward: the case for incentive salience. Psychopharmacology. 2007;191:391-431.

29. Berridge KC. From prediction error to incentive salience: mesolimbic computation of reward motivation. Eur J Neurosci. 2012;35:1124-43.

30. Zhang L, Dong Y, Doyon WM, Dani JA. Withdrawal from chronic nicotine exposure alters dopamine signaling dynamics in the nucleus accumbens. Biol Psychiatry. 2012;71:184-91.

31. Caggiula AR, Donny EC, White AR, Chaudhri N, Booth S, Gharib MA, et al. Cue dependency of nicotine self-administration and smoking. Pharmacol Biochem Behav. 2001;70:515-30.

32. Howe WM, Berry AS, Francois J, Gilmour G, Carp JM, Tricklebank M, et al. Prefrontal cholinergic mechanisms instigating shifts from monitoring for cues to cue-guided performance: converging electrochemical and fMRI evidence from rats and humans. J Neurosci. 2013;33:8742-52.

33. Brunzell DH, Mineur YS, Neve RL, Picciotto MR. Nucleus accumbens CREB activity is necessary for nicotine conditioned place preference. Neuropsychopharmacology. 2009;34:1993.

34. Madayag A, Lobner D, Kau KS, Mantsch JR, Abdulhameed O, Hearing M, et al. Repeated $\mathrm{N}$-acetylcysteine administration alters plasticity-dependent effects of cocaine. J Neurosci. 2007;27:13968-76.

35. Picciotto MR, Zoli M, Rimondini R, Léna C, Marubio LM, Pich EM, et al. Acetylcholine receptors containing the $\beta 2$ subunit are involved in the reinforcing properties of nicotine. Nature. 1998;391:173.

36. Kalivas PW. The glutamate homeostasis hypothesis of addiction. Nat Rev Neurosci. 2009;10:561.

37. Pistillo F, Clementi F, Zoli M, Gotti C. Nicotinic, glutamatergic and dopaminergic synaptic transmission and plasticity in the mesocorticolimbic system: focus on nicotine effects. Prog Neurobiol. 2015;124:1-27.

38. Picciotto MR, Mineur YS. Molecules and circuits involved in nicotine addiction: the many faces of smoking. Neuropharmacology. 2014;76:545-53.
39. Admon R, Kaiser R, Dillon D, Beltzer M, Goer F, Olson D, et al. Dopaminergic enhancement of striatal response to reward in major depression. Am J psychiatry. 2017; 174:378.

40. Knutson B, Fong GW, Adams CM, Varner JL, Hommer D. Dissociation of reward anticipation and outcome with event-related fMRI. Neuroreport. 2001;12: 3683-87.

41. Janes AC, Zegel M, Ohashi K, Betts J, Molokotos E, Olson D, et al. Nicotine normalizes cortico-striatal connectivity in non-smoking individuals with major depressive disorder. Neuropsychopharmacology. 2018;43:2445.

42. First MB, Spitzer RL, Gibbon M, Williams JB. Structured clinical interview for DSM-IV-TR axis I disorders, research version, patient edition. SCID-I/P. New York: Biometrics Research, New York State Psychiatric Institute; 2002.

43. Choi JH, Dresler CM, Norton MR, Strahs KR. Pharmacokinetics of a nicotine polacrilex lozenge. Nicotine Tob Res. 2003;5:635-44.

44. Benowitz NL, III PJ. Daily intake of nicotine during cigarette smoking. Clin Pharmacol Therapeutics. 1984;35:499-504.

45. Benowitz N. Systemic absorption and effects of nicotine from smokeless tobacco. Adv Dent Res. 1997;11:336-41.

46. Benowitz NL, Porchet $H$, Sheiner $L$, Jacob IIIP. Nicotine absorption and cardiovascular effects with smokeless tobacco use: comparison with cigarettes and nicotine gum. Clin Pharmacol Therapeutics. 1988;44:23-8.

47. Ziegler UE, Kauczok J, Dietz UA, Reith HB, Schmidt K. Clinical correlation between the consumption of nicotine and cotinine concentrations in urine and serum by competitive enzyme-linked immunosorbent assay. Pharmacology. 2004;72: 254-9.

48. Woolrich MW, Behrens TE, Beckmann CF, Jenkinson M, Smith SM. Multilevel linear modelling for FMRI group analysis using Bayesian inference. Neurolmage. 2004;21:1732-47.

49. Woo C-W, Krishnan A, Wager TD. Cluster-extent based thresholding in fMRI analyses: pitfalls and recommendations. Neurolmage. 2014;91:412-9.

50. Worsley $K$, Jezzard $P$, Matthews $P$, Smith S. Functional MRI: an introduction to methods. In: Jezzard P, Matthews PM, Smith SM. (eds). New York, NY: Oxford University Press. 2001. p. 251-70.

51. Pecina S, Berridge KC. Hedonic hot spot in nucleus accumbens shell: where do $\mu$-opioids cause increased hedonic impact of sweetness? J Neurosci. 2005;25:11777-86.

52. Smith KS, Berridge KC. Opioid limbic circuit for reward: interaction between hedonic hotspots of nucleus accumbens and ventral pallidum. J Neurosci. 2007;27:1594-605.

53. Chaudhri N, Caggiula AR, Donny EC, Booth S, Gharib M, Craven L, et al. Operant responding for conditioned and unconditioned reinforcers in rats is differentially enhanced by the primary reinforcing and reinforcement-enhancing effects of nicotine. Psychopharmacology. 2006;189:27-36.

54. Palmatier MI, Liu X, Matteson GL, Donny EC, Caggiula AR, Sved AF. Conditioned reinforcement in rats established with self-administered nicotine and enhanced by noncontingent nicotine. Psychopharmacology. 2007;195:235-43.

55. Overby PF, Daniels CW, Del Franco A, Goenaga J, Powell GL, Gipson CD, et al. Effects of nicotine self-administration on incentive salience in male Sprague Dawley rats. Psychopharmacology. 2018;235:1121-30.

56. Versaggi $\mathrm{CL}$, King CP, Meyer PJ. The tendency to sign-track predicts cue-induced reinstatement during nicotine self-administration, and is enhanced by nicotine but not ethanol. Psychopharmacology. 2016;233:2985-97.

57. Schultz W. Getting formal with dopamine and reward. Neuron. 2002;36:241-63.

58. Schultz W. Neuronal reward and decision signals: from theories to data. Physiol Rev. 2015;95:853-951.

59. Schultz W. Updating dopamine reward signals. Curr Opin Neurobiol. 2013; 23:229-38.

60. Reid MS, Mickalian JD, Delucchi KL, Hall SM, Berger SP. An acute dose of nicotine enhances cue-induced cocaine craving. Drug Alcohol Depend. 1998;49:95-104.

61. Horger BA, Giles MK, Schenk S. Preexposure to amphetamine and nicotine predisposes rats to self-administer a low dose of cocaine. Psychopharmacology. 1992;107:271-6.

62. Hogarth L, Chase HW. Parallel goal-directed and habitual control of human drugseeking: Implications for dependence vulnerability. J Exp Psychol: Anim Behav Process. 2011;37:261.

63. Hogarth L, Balleine BW, Corbit LH, Killcross S. Associative learning mechanisms underpinning the transition from recreational drug use to addiction. Ann N Y Acad Sci. 2013;1282:12-24.

64. Hu Y, Salmeron BJ, Gu H, Stein EA, Yang Y. Impaired functional connectivity within and between frontostriatal circuits and its association with compulsive drug use and trait impulsivity in cocaine addiction. JAMA Psychiatry. 2015;72:584-92.

65. Haber SN, Knutson B. The reward circuit: linking primate anatomy and human imaging. Neuropsychopharmacology. 2010;35:4-26.

66. Padoa-Schioppa C, Assad JA. Neurons in the orbitofrontal cortex encode economic value. Nature. 2006:441:223. 
The acute effects of nicotine on corticostriatal responses to distinct... KS Wang et al.

1214

67. Grabenhorst F, Rolls ET. Value, pleasure and choice in the ventral prefrontal cortex. Trends Cogn Sci. 2011;15:56-67.

68. Furl N, Averbeck BB. Parietal cortex and insula relate to evidence seeking relevant to reward-related decisions. J Neurosci. 2011;31:17572-82.

69. Lambert NM, McLeod M, Schenk S. Subjective responses to initial experience with cocaine: an exploration of the incentive-sensitization theory of drug abuse. Addiction. 2006;101:713-25.

70. Beier KT, Steinberg EE, DeLoach KE, Xie S, Miyamichi K, Schwarz L, et al. Circuit architecture of VTA dopamine neurons revealed by systematic input-output mapping. Cell. 2015;162:622-34.

71. Ferenczi EA, Zalocusky KA, Liston C, Grosenick L, Warden MR, Amatya D, et al. Prefrontal cortical regulation of brainwide circuit dynamics and reward-related behavior. Science. 2016;351:aac9698.

72. Taber MT, Das S, Fibiger HC. Cortical regulation of subcortical dopamine release: mediation via the ventral tegmental area. J Neurochemistry. 1995; 65:1407-10.

73. Hayden BY, Platt ML. Neurons in anterior cingulate cortex multiplex information about reward and action. J Neurosci. 2010;30:3339-46.

74. Lodge DJ. The medial prefrontal and orbitofrontal cortices differentially regulate dopamine system function. Neuropsychopharmacology. 2011;36:1227.

75. Wu J, Gao M, Shen J-X, Shi W-X, Oster AM, Gutkin BS. Cortical control of VTA function and influence on nicotine reward. Biochem Pharmacol. 2013;86: 1173-80.

76. Gaspar P, Berger B, Febvret A, Vigny A, Henry JP. Catecholamine innervation of the human cerebral cortex as revealed by comparative immunohistochemistry of tyrosine hydroxylase and dopamine-beta-hydroxylase. J Comp Neurol. 1989;279: 249-71.

77. Emson P, Koob G. The origin and distribution of dopamine-containing afferents to the rat frontal cortex. Brain Res. 1978;142:249-67.

78. Salamone JD. The involvement of nucleus accumbens dopamine in appetitive and aversive motivation. Behavioural Brain Res. 1994;61:117-33.

79. Marshall DL, Redfern PH, Wonnacott S. Presynaptic nicotinic modulation of dopamine release in the three ascending pathways studied by in vivo microdialysis: comparison of naive and chronic nicotine-treated rats. J Neurochemistry. 1997;68:1511-9.

80. Nisell M, Nomikos GG, Hertel P, Panagis G, Svensson TH. Condition-independent sensitization of locomotor stimulation and mesocortical dopamine release following chronic nicotine treatment in the rat. Synapse. 1996;22:369-81.

81. Amiez C, Joseph JP, Procyk E. Anterior cingulate error-related activity is modulated by predicted reward. Eur J Neurosci. 2005;21:3447-52.

82. Kennerley SW, Behrens TE, Wallis JD. Double dissociation of value computations in orbitofrontal and anterior cingulate neurons. Nat Neurosci. 2011;14:1581.

83. Brown JW, Braver TS. Learned predictions of error likelihood in the anterior cingulate cortex. Science. 2005;307:1118-21.

84. Hart AS, Rutledge RB, Glimcher PW, Phillips PE. Phasic dopamine release in the rat nucleus accumbens symmetrically encodes a reward prediction error term. J Neurosci. 2014;34:698-704.

85. Pagnoni G, Zink CF, Montague PR, Berns GS. Activity in human ventral striatum locked to errors of reward prediction. Nat Neurosci. 2002;5:97.

86. Day JJ, Roitman MF, Wightman RM, Carelli RM. Associative learning mediates dynamic shifts in dopamine signaling in the nucleus accumbens. Nat Neurosci. 2007; 10:1020.

87. Wylie KP, Rojas DC, Tanabe J, Martin LF, Tregellas JR. Nicotine increases brain functional network efficiency. Neurolmage. 2012;63:73-80.

88. Falcone M, Cao W, Bernardo L, Tyndale RF, Loughead J, Lerman C. Brain responses to smoking cues differ based on nicotine metabolism rate. Biol Psychiatry. 2016;80:190-7.

89. Haney M, Bedi G, Cooper ZD, Glass A, Vosburg SK, Comer SD, et al. Predictors of marijuana relapse in the human laboratory: robust impact of tobacco cigarette smoking status. Biol Psychiatry. 2013;73:242-8.

90. Fluharty M, Taylor AE, Grabski M, Munafò MR. The association of cigarette smoking with depression and anxiety: a systematic review. Nicotine Tob Res. 2016;19:3-13.

91. Taylor G, McNeill A, Girling A, Farley A, Lindson-Hawley N, Aveyard P. Change in mental health after smoking cessation: systematic review and meta-analysis. BMJ. 2014;348:g1151. 